\title{
Postgraduate education in spine surgery: the blended online learning concept
}

\author{
Robert Gunzburg ${ }^{1} \cdot$ Marek Szpalski $^{2} \cdot$ Claudio Lamartina $^{3}$
}

Received: 29 July 2018 / Accepted: 30 July 2018 / Published online: 6 August 2018

c) Springer-Verlag GmbH Germany, part of Springer Nature 2018

In February 2015, an interview about online learning and education with Prof. L.R. Reif, Principal of the MIT in Boston, was published in the "Neue Zürcher Zeitung," one of Europe's leading newspapers. In this interview, Prof. Reif made some strong statements: "1. Online learning has initiated a revolution and is the most important innovation in education since the introduction of the printed word by Gutenberg in the 16th century. 2. Universities as well as teaching institutions and educational organizations and tools such as specialist societies and scientific journals, which do not keep up, will be left behind."

The last 5-8 years saw an increasing number of online educational programs in a wide field of undergraduate (College) and graduate (University) programs as well as in non-academic educational programs. According to the US National Center for Education Statistics (www.nces.ed.gov) in 2012, 2.6 million college students were learning without setting a foot in an actual classroom and more than 2.8 million students were taking at least some of the classes digitally (blended learning). In the past 6 years, close to 800 universities have created over 8000 Massive Open Online Courses, and in only the three first months of 2018 over 200 Universities have introduced about 600 free or at least partially free online courses. (http://www.worldnetexchange.eu/ news/200-universities-just-launched-600-free-online-cours es-heres-the-full-list/).

Originally those MOOCS, i.e., the teaching videos as well as the certificates were available free of charge, the financing of these programs being provided by private donations

Robert Gunzburg

robert@gunzburg.be

1 Cavell Spine Centre, Edith Cavell Clinic, Edith Cavell Street 32, 1180 Brussels, Belgium

2 Department of Orthopedics, Iris South Hospital, 21 Avenue Coghen, 1180 Brussels, Belgium

3 II Spine Surgery Division, IRCSS Istituto Ortopedico Galeazzi, Via Riccardo Galeazzi, 4, 20161 Milan, Italy and organizations like the Bill Gates Foundation and others, as well as by crowdfunding. As the providers of those programs needed funding for further development and distribution, they started to put at least some parts of the program (e.g., acquirement of the certificate) under a cost scheme by developing business models to sustain these educational programs. The most popular business model today is the tuition-sharing model, with free tuition by the educational institution, which provides content and study guidance, and a charge by the tech company, which provides the learning technology. Indeed, the tech companies have to do upfront investments in order to design and finally run the programs with rapidly developing IT technologies. As an example, Lecturio (https://www.lecturio.com), the first online Medical School Platform which originally started in Germany and is now expanding to the USA, is a platform, which is a combination of a guided digital library and online lectures with MCQ specifically designed for the preparation of the different exams throughout Medical School. As of today more than 20,000 students have enrolled in the Lecturio program paying (reasonable) fees.

Some major e-learning platforms have been founded by Ivy League Universities, the best example is EDX, (www. edx.org) created by Harvard University and Massachusetts Institute of Technology in 2012. Today over 50 major universities from USA, Europe and Asia Pacific offer over 1900 courses in several languages to which over 14 million learners enlisted worldwide for over 52 million courses enrollments. Courses are free for individuals but payment is required for certification as well as for group courses purchased by businesses. In addition to the basic courses, some universities offer the so-called paying "MicroMasters" which can accelerate obtention of a master's degree.

Common to all these programs is flexibility of learning: Students can learn whenever they want and where they want. They can listen to lectures as many times they wish, and there is easy access to additional knowledge sources. This leads to an efficient time management in the learning 
process; the only thing needed is an Internet connection. Such functionality can be described as learning on-demand. A College or University is no longer limited to physical classrooms and can reach limitless students worldwide. This leads to globalization of standardized, comprehensive and updated knowledge and signifies a true democratization of knowledge.

Most of the above-mentioned programs are the so-called blended educational programs, i.e., the student has the option to acquire basic knowledge online, and for the application of the knowledge in the real world, face-to-face meetings are offered, where educator-student as well as student-student interactions can take place both live and virtually.

Interestingly enough, as online learning, respectively blended learning, is today getting more and more an integral part of any educational effort, it has not yet penetrated postgraduate education in medical specialties. Yet, the demand of documented professional qualification for physicians and surgeons is increasingly required by hospital organizations, employers of specialist doctors, as well as by health and malpractice insurances and, last but not least, by patient organizations.

In medicine, specifically in spine surgery, we are far away from such programs, although a lot of effort has been made by many different organizations to structure a postgraduate education in spine surgery. The extensive information which is digitally available today on different platforms is generally unstructured and not comprehensive. What is more, the acquisition of this knowledge is not measured by an objective examination. It is somewhat difficult to fathom why medical doctors in postgraduate training, specifically surgeons, who have busy schedules and a need of presence availability, would not be among the first to be keen to register for a cost-effective, online blended education program. This would eliminate the need to travel to course locations with all the time involved and expenses for travel and lodging. Of course, there is the attraction of social interaction between peers and between and with teachers, but blended education programs offer these as well.

There is, unfortunately, still no such a thing as a European spine surgery certification. The continuous medical education (CME) points given by organizations that offer educational programs play only a marginal role toward qualification as a spine surgery specialist. A blended online learning program offered by an international faculty and measured by an examination at the end, however, could lead to certification.

The European Spine Journal as one of the leading international Spine Specialist Journals recognized the new trends in education as early as in 2014. This lead to the creation of the post of Deputy-Editor for web-based education which was entrusted to Max Aebi, founder and past Editor-in-Chief, who had already been instrumental in this field by launching the successful open operation theater (OOT) online video program. Indeed, peer-reviewed journals that are responsible for publishing new scientific information are nowadays also involved in continuous and postgraduate education and are gradually mutating to digital knowledge transfer platforms.

Other journals worldwide also recognize this need for e-learning and the necessity to search for experienced partners. The Journal of Bone and Joint Surgery (Am) partners with Miller Review Courses (MRC) (www.millerreview.org) to propose a virtual recertification course (https://purchase. jbjs.org/virtual-recertification-course/virtual-recertification -course.html) preparing, and giving the required CME credits, for the American Board of Orthopedic Surgery (www. abos.org) recertification examination. In this joint-venture MR., a company active for 25 years, is obviously providing JBJS its expertise in decades of orthopedic courses and JBJS is providing its online platform and orthopedic marketing power.

The ABOS itself, which is currently organizing its recertification examinations exclusively at test centers (with oral or multiple-choice examination options), is moving toward an online system. Beginning in 2019, ABOS will introduce a third option for the examination called "Web-Based Longitudinal Assessment Program (ABOS WLA)" which will be a yearly "open book" assessment based on knowledge sources which will be available on the ABOS website. (https://www. abos.org/moc/examination-options.aspx).

The American Board of Neurological Surgeons, which certifies and re-certifies neurosurgeons, already employs such an e-learning and e-examination continuous assessment system which totally replaces "physical" re-certification examination, the "ABNS MOC Part III Learning Tool." (https://www.abns.org/moc-part-iii-learning-tool/).

During the last four years, a blended Postgraduate Education Program in Spine Surgery, eccElearning, was created by Max Aebi with the help of a dedicated group of more than 140 well-known international experts. The program consists of lectures and instructional videos organized in nine modules representing basically a "living" book. To each module belongs a face-to-face case-based tutorial where the acquired theoretical knowledge is applied to real cases in group work under the guidance of clinically experienced moderators. Each module is followed by a multiple-choice evaluation. The platform has recently been awarded 500 CME points by the UEMS in Brussels for the lecture part only, so this type of e-learning platform can both serve as a spine qualification learning tool as well as a continuous education system. The well-known international faculty involved in this spinal e-learning platform is of high scientific and clinical caliber, and after thorough content reviewing, the Editors deemed it appropriate to endorse the quality of the scientific content of eccElearning, of which more information can be found at www.eccElearning.com. 
The digitalization and the on-demand character of "anytime, anywhere and as often as needed," which is rapidly penetrating and dominating our daily life, has started to become also an integral part of postgraduate as well as continuous specialist education and will allow to establish a standardized, comprehensive and complete expertise, independent from the location and the local circumstances of the learner.

\section{Compliance with ethical standards}

Conflict of interest The authors declare that they have no conflict of interest. 